\title{
A Practical, One-Clinic Visit Protocol for Pharmacokinetic Profile Generation with the ADVATE myPKFiT Dosing Tool in Severe Hemophilia A Subjects
}

\author{
Victor S. Blanchette ${ }^{1,2}$ Laura Zunino ${ }^{3}$ Viviane Grassmann ${ }^{3}$ Chris Barnes ${ }^{4,5}$ Manuel D. Carcao ${ }^{1,3}$ \\ Julie Curtin ${ }^{6,7}$ Shannon Jackson ${ }^{8}$ Liane Khoo ${ }^{9}$ Vladimir Komrska ${ }^{10}$ David Lillicrap ${ }^{11}$ \\ Massimo Morfini ${ }^{12}$ Gabriela Romanova ${ }^{13,14}$ Derek Stephens ${ }^{15}$ Ester Zapotocka ${ }^{10}$ \\ Margaret L. Rand ${ }^{1,16,17,18,19}$ Jan Blatny ${ }^{14,20}$
}

${ }^{1}$ Division of Haematology/Oncology, The Hospital for Sick Children, Toronto, Ontario, Canada

${ }^{2}$ Department of Paediatrics, University of Toronto, Toronto, Ontario, Canada

${ }^{3}$ Child Health Evaluative Sciences, Research Institute, The Hospital for Sick Children, Toronto, Ontario, Canada

${ }^{4}$ Haematology Department, The Royal Children's Hospital Melbourne, Victoria, Australia

${ }^{5}$ Haematology Research, Murdoch Children's Research Institute, Victoria, Australia

${ }^{6}$ Department of Haematology, The Children's Hospital at Westmead, Sydney, Australia

${ }^{7}$ Department of Paediatrics and Child Health, University of Sydney, Sydney, Australia

${ }^{8}$ Division of Haematology, St. Paul's Hospital, Vancouver, British Columbia, Canada

${ }^{9}$ Haematology Department, Royal Prince Alfred Hospital, NSW Health Pathology, Sydney, Australia

10 Department of Paediatric Haematology and Oncology, University Hospital Motol, Prague, Czech Republic

${ }^{11}$ Department of Pathology and Molecular Medicine, Queen's University, Kingston, Ontario, Canada
Address for correspondence Victor S. Blanchette, MB BChir, $\operatorname{FRCP}(C)$, FRCP, Division of Hematology/Oncology, The Hospital for Sick Children, 555 University Avenue, Toronto, ON, M5G1X8, Canada (e-mail: victor.blanchette@sickkids.ca).

12 Italian Association of Haemophilia Centres, Florence, Italy

${ }^{13}$ Department of Clinical Haematology, University Hospital Brno, Brno, Czech Republic

${ }^{14}$ Faculty of Medicine, Masaryk University, Brno, Czech Republic

15 Department of Clinical Research Services, The Hospital for Sick Children, Toronto, Canada

${ }^{16}$ Translational Medicine, Research Institute, The Hospital for Sick Children, Toronto, Canada

17 Department of Laboratory Medicine and Pathobiology, University of Toronto, Toronto, Ontario, Canada

${ }^{18}$ Department of Biochemistry, University of Toronto, Toronto, Ontario, Canada

${ }^{19}$ Department of Paediatrics, University of Toronto, Toronto, Ontario, Canada

20 Department of Paediatric Haematology, University Hospital Brno, Brno, Czech Republic

\section{Abstract \\ Keywords \\ - factor VIII \\ - hemophilia A \\ - pharmacokinetic \\ - observational study \\ - population PK}

Standard pharmacokinetic (PK) assessments are demanding for persons with hemophilia $A$, requiring a 72-hour washout and 5 to 11 timed blood samples. A no-washout, single-clinic visit, sparse sampling population PK (PPK) protocol is an attractive alternative. Here, we compared PK parameters obtained with a traditional washout, 6-sampling time point PPK protocol with a no-washout, single-clinic visit, reverse 2sampling time point PPK protocol in persons with severe hemophilia $A(S H A)$ receiving ADVATE. A total of 39 inhibitor-negative males with SHA (factor VIII activity [FVIII:C] $<2 \%$ ) were enrolled in a prospective sequential design PK study. Participants completed a washout, 6-sampling time point PPK protocol as well as a no-washout, reverse 2-sampling time point protocol, with samples taken during a single 3-hour clinic visit 24 hours post home infusion of FVIII and then 3 hours post infusion in clinic. FVIII:C levels were analyzed by received

July 8,2020

accepted after revision

January 23, 2021

published online

January 27, 2021
DOI https://doi.org/ 10.1055/a-1376-0970. ISSN 0340-6245.
(C) 2021. The Author(s).

This is an open access article published by Thieme under the terms of the Creative Commons Attribution-NonDerivative-NonCommercial-License, permitting copying and reproduction so long as the original work is given appropriate credit. Contents may not be used for commercial purposes, or adapted, remixed, transformed or built upon. (https://creativecommons.org/ licenses/by-nc-nd/4.0/) Georg Thieme Verlag KG, Rüdigerstraße 14, 70469 Stuttgart, Germany 
one-stage and chromogenic assays; blood group and von Willebrand factor antigen (VWF:Ag) were determined; and PK parameters were analyzed using the ADVATE myPKFiT dosing tool. There was moderate to almost perfect agreement for the PK parameters obtained with the 2- and the 6- point PPK protocols using a one-stage FVIII:C assay and a substantial to almost perfect agreement using a chromogenic FVIII:C assay. Significant associations between specific PK parameters and blood group and VWF:Ag were observed. The no-washout, single-clinic visit, reverse 2-sampling time point PPK protocol can be used in the routine clinical setting since it demonstrates sufficient accuracy compared with the more demanding and less practical washout, 6-sampling time point PPK protocol in persons with SHA receiving ADVATE.

\section{Introduction}

Regular replacement therapy ("prophylaxis") with clotting factor concentrates has been demonstrated to reduce the frequency of joint bleeds (hemarthroses) in individuals with moderate/severe hemophilia $\mathrm{A}$ and $\mathrm{B}$, resulting in the prevention of disabling and painful joint damage and improved health outcomes. ${ }^{1}$

There is evidence that the risk of spontaneous joint bleeding in persons with hemophilia A increases with the duration of time spent with factor VIII activity (FVIII:C) levels below 1 international unit (IU)/dL (1\%). ${ }^{2}$ As such, the recommendation has been to maintain FVIII:C levels $>1 \%$ for persons with severe hemophilia. Factor levels (peaks and troughs) in persons with severe hemophilia A receiving prophylaxis are primarily influenced by the individual's pharmacokinetic (PK) profile together with the frequency of FVIII infusions. In clinical practice, FVIII doses are typically calculated based on body weight without adjustment for individual PK profiles between individuals with severe hemophilia A. ${ }^{3}$ This may result in vastly different levels of protection between individuals when given similar doses on a per weight basis; those with a "better" PK profile, as evidenced by a slower clearance $(\mathrm{Cl})$ of infused FVIII and a longer terminal half-life $\left(t_{1 / 2}\right)$, may be well protected while those with a "worse" PK profile, as evidenced by a faster $\mathrm{Cl}$ of infused FVIII and a shorter $t_{1 / 2}$, may be inadequately protected. To optimize treatment, there has been an increased interest in individualized PK-based dosing.

Traditional PK assessment for persons with moderate/ severe hemophilia $A$, however, requires a long washout period (minimum 72 hours) and a total of 5 to 11 blood samples drawn at specific time points following an infusion of FVIII with the subject in a nonbleeding state. ${ }^{4,5}$ This is demanding for both patients/families and health care professionals alike. ${ }^{6}$

In 1977, Sheiner et al proposed the population PK (PPK) model (also known as nonlinear mixed effects analysis) that has recently become a popular approach used by PK experts. $^{7-9}$ FVIII PPK models have been developed using individual PK measurements from a defined population of persons with hemophilia $A$ and covariates such as age, weight (or lean body mass/fat-free mass), and von Willebrand factor antigen (VWF:Ag) levels. ${ }^{10}$ Bayesian forecast- ing, a method of inference using Bayes' theorem to update probabilities as information becomes available, is used to generate PK parameters using PPK models. Most importantly, these models allow PK analysis to be accomplished without mandatory washouts and with sparse blood sampling (2-4 timed blood samples) from the subject under study. $^{11,12}$

The principal aim of this study was to compare two PK protocols for persons with severe hemophilia A receiving ADVATE: (1) a more traditional, 6-sampling time point PK protocol with a minimum 72-hour washout and (2) a more practical, single clinic visit "reverse" 2 -sampling time point PK protocol with no-washout. The latter was achieved during a single 3-hour outpatient hemophilia clinic visit, with the first sample drawn upon arrival to the clinic, 24 hours following infusion of a regular prophylaxis dose in the patient's home. Immediately after this sample is drawn, the patient receives an administration of FVIII (in clinic), and then 3 hours postinfusion a drawing of the second sample is done.

The following PK parameters were determined: $\mathrm{Cl} ; t_{1 / 2}$; volume of distribution at steady state $\left(V_{\mathrm{ss}}\right)$; and time to FVIII:C of $1 \%$ above baseline (tt $1 \%)$. Our working hypothesis was that a "user-friendly" (single outpatient hemophilia clinic visit, sparse blood sampling), no-washout PK protocol would yield PK profiles that would be comparable to the more demanding (multiple clinic visits, more frequent blood samples) washout PK protocol. If confirmed, this could set the stage for more widespread use of individualized, PK-guided prophylaxis in persons with hemophilia. ${ }^{13}$ This study also afforded us the opportunity to reinforce the previously described influence of blood group and VWF:Ag levels on PK parameters, and to confirm the large interindividual variability that exists in PK parameters. The results of our international collaborative PK study are detailed in this communication.

\section{Methods}

\section{Study Design}

Seven hemophilia treatment centers participated in this international, multicenter, prospective sequential design study: The Hospital for Sick Children (Toronto, Canada); St. Paul's Hospital (Vancouver, Canada); University Hospital Brno (Brno, 
Czech Republic); University Hospital Motol (Prague, Czech Republic); Royal Children's Hospital (Melbourne, Australia); Royal Prince Alfred Hospital (Sydney, Australia); and The Children's Hospital at Westmead (Sydney, Australia).

In this observational study, all participants were required to complete a 6- and a 2-point PK protocol (see below) within 6 months of enrollment. Depending on participant preference, PK blood samples were taken from a peripheral vein or an indwelling peripheral or central venous access device (e.g., a port-a-catheter) following the recommended protocol for blood discards (1-2 mL for an indwelling peripheral cannula, 5-7 $\mathrm{mL}$ for a port-a-catheter).

Blood samples were collected into $3.2 \%$ sodium citrate tubes and processed locally within 3 hours of collection, centrifuging at $2,500 \times \mathrm{g}$ for 15 minutes at room temperature and aliquoting the resulting platelet-poor plasma into cryovials for storage at $-20^{\circ} \mathrm{C}$ for a maximum of 48 hours before transferring to $-80^{\circ} \mathrm{C}$. Frozen samples were shipped on dry ice to the central reference coagulation laboratory (Department of Pathology and Molecular Medicine, Queen's University, Kingston, Canada) for analysis.

\section{6-Sampling Time Point PK Protocol}

Participants were required to undergo a minimum washout of 72 hours to ensure a low baseline measure of FVIII:C. They were infused intravenously with approximately $50 \mathrm{IU} / \mathrm{kg}$ body weight rounded to the closest full vial size of a standard halflife (SHL) recombinant full-length FVIII concentrate (rFVIII; ADVATE; Baxalta US Inc., a Takeda company, Lexington, Massachusetts, United States) in clinic. Blood samples were taken at 6 time points: $<30$ minutes preinfusion; and 1 hour \pm 5 minutes, 3 hours \pm 15 minutes, 9 hours \pm 1 hour, 24 hours \pm 2 hours, and 48 hours \pm 2 hours postinfusion (-Fig. 1). This protocol, requiring a washout and six samples, was designed as a reference protocol for the more practical two-sample single-clinic visit protocol without a washout (see the following).

\section{6-point PK protocol}

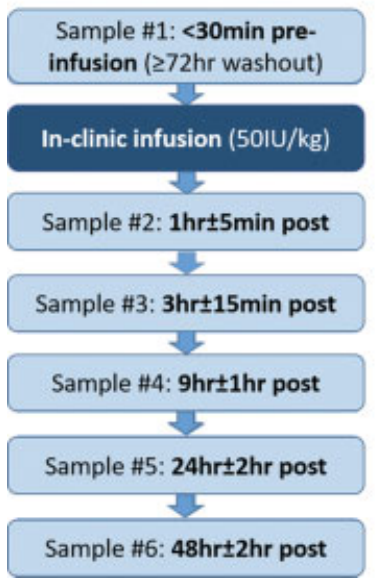

Fig. 1 Flowchart of the 6-point and 2-point pharmacokinetic (PK) protocols.

\section{Reverse 2-Sampling Time Point PK Protocol}

This protocol consisted of two blood samples collected during a single-clinic visit with no washout. The first blood sample was drawn in clinic 24 hours \pm 2 hours after the participant infused their regular prophylactic dose of rFVIII (ADVATE) at home (15-50 IU $/ \mathrm{kg}$ ); then, following an infusion of approximately $25 \mathrm{IU} / \mathrm{kg}$ rFVIII (ADVATE; rounded to the nearest full vial size) administered at the time of the first blood draw in clinic, a postinfusion sample was taken at 3 hours \pm 15 minutes (-Fig. 1). As such, the 2-sampling point PK protocol could be accomplished in one 3-hour outpatient clinic visit. This timeframe was chosen as a routine clinic visit that includes medical/nursing, physiotherapy, and psychosocial assessments and possible other blood work takes, in our experience, approximately 3 hours to complete.

\section{Participant Selection Criteria}

Both adults and children were included in this study, with no minimum or maximum age cut-offs. Inclusion criteria included a confirmed diagnosis of severe hemophilia $\mathrm{A}$, defined in this study as a baseline FVIII:C level of $<2 \%$; participant receiving ADVATE for prophylaxis; and body weight $\geq 12 \mathrm{~kg}$ and $\leq 120 \mathrm{~kg}$. Exclusion criteria included presence of an inhibitor to FVIII ( $\geq 0.6 \mathrm{BU} / \mathrm{mL}$ using the Nijmegen modification of the Bethesda assay); a history of recent events that might affect FVIII half-life (e.g., infection, significant bleed, surgery or an invasive procedure) within 2 weeks of blood sampling; human immunodeficiency virus (HIV) positivity with a CD4 count $<200$ cells $/ \mu \mathrm{L}$; or significant hepatic dysfunction defined as alanine aminotransferase or aspartate aminotransferase levels $>5 \times$ the upper limit of normal. The last two criteria were used to ensure that participants had no laboratory evidence of clinically severe hepatitis or HIV-associated immunodeficiency, comorbidities that might impact on the PK handling of FVIII. Inhibitor status for participants was determined according to the two most recent, consecutive inhibitor levels on record prior to study enrolment.

Written informed consent was obtained from each participant and/or their parents/legal guardians (as appropriate); local research ethics boards approved the study. The study was registered on ClinicalTrials.gov (NCT02750085).

\section{Laboratory Assays}

Plasma samples were assayed in a central reference coagulation laboratory (Kingston, Canada) using one-stage and chromogenic FVIII:C assays. The Siemens BCS XP system (Marburg, Germany) was used for both assays, and all reagents used were from Siemens. Standard human plasma (ORKL17) was used to generate standard curves. FVIIIdeficient plasma (OTXW17), Owren's veronal buffer (B4237-23), $\mathrm{CaCl}_{2}$ (ORHO37), and Actin FS (B4218-100; activating material ellagic acid) were used for the one-stage assays. The FVIII chromogenic assay kit (B4238-40) containing reagents FX, FIX, substrate, substrate buffer, and Owren's veronal buffer were used for the chromogenic FVIII:C assay. One-stage FVIII:C assays were also performed on plasma samples in the routine clinical coagulation laboratories of study sites as per their local protocols. 
VWF:Ag levels were measured by enzyme-linked immunosorbent assay (ELISA) using polyclonal rabbit anti-human VWF-coating antibody (Ab) (A0082; Dako, Glostrup, Denmark), polyclonal rabbit anti-human VWF/HRP detecting Ab (P0026; Dako), and Cryocheck Normal Reference Plasma (CCNRP-05; Precision BioLogics, Dartmouth, Canada). VWF propeptide (VWF:pp) levels were measured by ELISA using Ab pair anti-human VWF:pp MW1939 (Antibody Chain, Utrecht, Netherlands). The reference plasma used for the VWF:pp assay was Cryocheck Normal Reference Plasma. FVIII non-neutralizing Ab levels were measured by ELISA. ADVATE was used as a coating material, human anti-human FVIII monoclonal Ab EL-14 as reference Ab (kindly provided by Jan Voorberg, Sanquin, University of Amsterdam, the Netherlands), and goat anti-human IgG-HRP as detecting Ab (2040-05; Southern Biotech, Birmingham, United States).

\section{Pharmacokinetic Parameters and Analysis}

The PK parameters reported herein were chosen in accordance with the guidelines from the FVIII and FIX Subcommittee of the Scientific and Standardization Committee of the International Society on Thrombosis and Haemostasis (ISTH). ${ }^{4}$ They include $\mathrm{Cl}\left(\mathrm{mL} / \mathrm{h} / \mathrm{kg}\right.$ ); $t_{1 / 2}$ (hours); $V_{\mathrm{ss}}$ (dL/kg); and tt $1 \%$ (hours). $t_{1 / 2}$ is defined as the time required for the FVIII:C level to decrease by $50 \%$ after the initial phase of distribution is completed, and thus reflects drug elimination. ${ }^{14}$

PK parameters from the 6-point PK were calculated using Phoenix WinNonlin 7.0 (Certara USA, Inc.; Princeton, United States), considered the gold standard for analyzing individual PK data, and ADVATE myPKFiT v. 3.1 (hereafter referred to as myPKFiT; Baxalta U.S. Inc., a Takeda company, Lexington, Massachusetts, United States). PK parameters from the 2-point PK were calculated only using the myPKFiT dosing tool.

PK analysis using WinNonlin is based on a two-compartment model with single bolus input using first-order output and micro-constants as primary parameters. The myPKFiT dosing tool was built from the ADVATE pre-licensure PK data using 184 full PK datasets (10 postinfusion samples) from 100 adults/adolescents with hemophilia A (10-65 years of age) and 52 reduced sample PK datasets (four postinfusion samples) from 52 boys with hemophilia A (1-6 years of age). ${ }^{15,16}$ The myPKFiT Web-based dosing tool is based on a Bayesian algorithm that assumes a two-compartment model for ADVATE as best fit for data and uses four basic parameters $(\mathrm{Cl}$; volume of distribution [V1]; volume of the second compartment [V2]; and intercompartmental $\mathrm{Cl}[Q])$. The model demonstrates good agreement between model predictions and observations of FVIII levels. Interindividual variance, as well as the covariates of age and body weight, are incorporated into the model. The model and its development are detailed in Björkman et al. ${ }^{11}$

\section{Statistical Analyses}

Statistical analyses were conducted using MedCalc Statistical Software version 18.10.2 (MedCalc Software; Ostend, Belgium). Intraclass correlations (ICCs) were used to assess the agreement of myPKFiT PK parameters obtained with the 6-point and 2-point PK protocols. The agreement of PK parameters of the 6-point protocol between WinNonlin and myPKFiT was also calculated using ICCs. The ICC values are interpreted as: $>0.8$, almost perfect; 0.6 to 0.8 , substantial; 0.4 to 0.6 , moderate; 0.2 to 0.4 , fair; 0.01 to 0.2 , slight; and $<0.01$, poor agreement. ${ }^{17,18}$ Pearson correlation coefficients were used to determine the extent of linear correlation between PK parameters and (1) VWF:Ag; (2) VWF:pp; (3) VWF:pp/Ag ratio; and (4) age. Pearson correlation coefficient values are interpreted as: 1 , perfect; 0.9 to 0.8 , very strong; 0.7 to 0.6 , moderate; 0.5 to 0.3 , fair; 0.2 to 0.1 , poor; and 0 , no agreement. A negative value means that the variables are inversely related. ${ }^{19}$

Paired $t$-tests were used to compare FVIII:C levels from the one-stage and chromogenic FVIII:C assays, and one-way analysis of variance (ANOVA) was used for comparing VWF:Ag levels in $\mathrm{O}$ versus non-O blood group subjects. Results from paired $t$-tests and one-way ANOVA are presented as means \pm standard deviations. Postinfusion FVIII:C levels were corrected using the Björkman formula, FVIII = FVIII * $(1-$ [baseline/ $\left.C_{\text {max }}\right]$ ). ${ }^{20}$ Descriptive statistics, i.e., means, medians, standard deviations, and confidence intervals, were used to summarize data, and $p$-values are presented where appropriate. A $p$-value $<0.05$ was considered to be statistically significant. A sample size of 35 patients was deemed necessary to achieve $80 \%$ power to detect an ICC of 0.8 under the alternative hypothesis, when the ICC under the null hypothesis is 0.5 using an F-test with a significance level of 0.01 .

\section{Results}

Thirty-nine males (median age: 11 years; range: 2-69 years) with severe hemophilia A (FVIII:C $<1 \%, n=31$; FVIII:C: 1 to $<2 \%, n=8$ ) participated in this study. Twenty-nine of the 39 study subjects were of age 18 years or less at the time of study. Characteristics of the study group are summarized in - Table 1.

PK parameters obtained with the WinNonlin program (two-compartment model) and the myPKFiT dosing tool were compared using both one-stage and chromogenic FVIII:C levels from the washout, 6-point PK protocol. An almost perfect agreement was observed when comparing $\mathrm{Cl}$ ( 0.95 and 0.94 , respectively) and $V_{\text {ss }}(0.81$ and 0.81 , respectively) using one-stage and chromogenic FVIII:C results.

\section{Comparison between the 6-Point and 2-Point PK Protocols}

The PK parameters of $\mathrm{Cl}$ and $t_{1 / 2}$, calculated using the myPKFiT dosing tool for the (washout) 6-point and the (no washout) single-clinic visit 2-point PK protocols using both one-stage and chromogenic FVIII:C levels (determined in the central laboratory), are shown in - Fig. 2 as spaghetti plots connecting single subjects (all participants, those with 0 blood group, and those with non-O blood group). For all participants, the agreement between the protocols for $\mathrm{Cl}$ was substantial and moderate using the one-stage and chromogenic FVIII:C levels, respectively, and for $t_{1 / 2}$, it was almost perfect using both assays (-Table $\mathbf{2}$ ).

For both $\mathrm{Cl}$ and $t_{1 / 2}$, there was an approximate three- to five-fold difference between the lowest and highest values obtained with the one-stage and chromogenic FVIII:C assays for both the 6- and 2-point PK protocols (-Fig. 3). 
Table 1 Characteristics of the study cohort

\begin{tabular}{|c|c|c|c|c|}
\hline & \multicolumn{2}{|l|}{ Pediatric } & \multirow[t]{2}{*}{ Adult ( $>18 \mathrm{y}$ ) } & \multirow[t]{2}{*}{ Total $^{\mathrm{a}}$} \\
\hline & $(<12 \mathrm{y})$ & $(12-18 y)$ & & \\
\hline Sample size $(n)$ & 20 & 9 & 10 & 39 \\
\hline Age $(y)$ & $6(2-11)$ & $14(13-17)$ & $28.5(22-69)$ & $11(2-69)$ \\
\hline Weight $(\mathrm{kg})$ & $23.6(13.5-53.0)$ & $70.4(43.8-77.6)$ & $83.5(74.0-105.0)$ & $48.4(13.5-105.0)$ \\
\hline \multirow[t]{5}{*}{ Blood group (\%) } & A (35.0\%) & $\mathrm{A}(33.3 \%)$ & A (50.0\%) & $\mathrm{A}(38.5 \%)$ \\
\hline & B (20.0\%) & $\mathrm{B}(0 \%)$ & $\mathrm{B}(0 \%)$ & B (10.3\%) \\
\hline & $\mathrm{AB}(0 \%)$ & $\mathrm{AB}(11.1 \%)$ & $\mathrm{AB}(20.0 \%)$ & $\mathrm{AB}(7.7 \%)$ \\
\hline & $\mathrm{O}(40.0 \%)$ & $\mathrm{O}(44.5 \%)$ & $\mathrm{O}(20.0 \%)$ & $\mathrm{O}(35.9 \%)$ \\
\hline & Unknown (5.0\%) & Unknown (11.1\%) & Unknown (10.0\%) & Unknown (7.7\%) \\
\hline VWF:Ag ${ }^{\mathrm{b}}(\mathrm{IU} / \mathrm{mL})$ & $0.8(0.4-1.3)$ & $1.0(0.5-1.7)$ & $1.0(0.5-1.6)$ & $0.8(0.4-1.7)$ \\
\hline VWF:pp ${ }^{\mathrm{b}}(\mathrm{IU} / \mathrm{mL})$ & $1.1(0.6-3.5)$ & $1.1(0.8-1.7)$ & $1.1(0.8-2.3)$ & $1.1(0.6-3.5)$ \\
\hline VWF:pp/VWF:Ag ${ }^{b}$ & $1.4(0.7-4.5)$ & $1.0(0.6-2.7)$ & $1.0(0.9-2.1)$ & $1.2(0.6-4.5)$ \\
\hline
\end{tabular}

Abbreviations: VWF:Ag, von Willebrand factor antigen; VWF:pp, von Willebrand factor propeptide.

Note: Values shown are medians with ranges shown in parenthesis.

${ }^{\mathrm{a}}$ All cases were negative for non-neutralizing FVIII binding antibodies ( $\mathrm{lgG}$ ) except for four subjects who had low-level positive results.

bLevels were measured after a 72-hour washout. The total sample size for these variables is 35 . No samples were available for analysis for four patients, all from the same hemophilia treatment center.

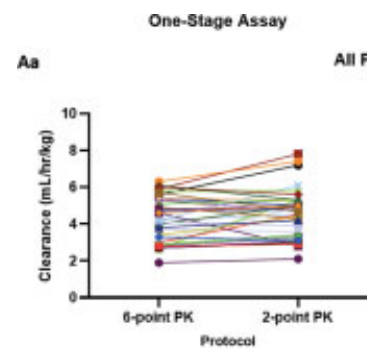

Ab

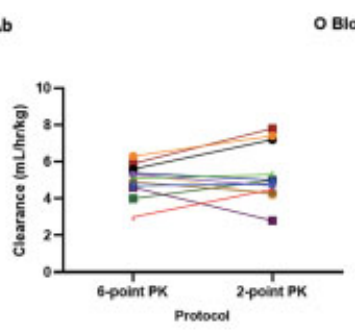

Ac

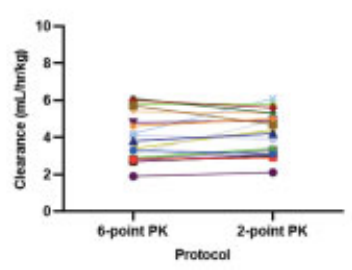

O Blood Group

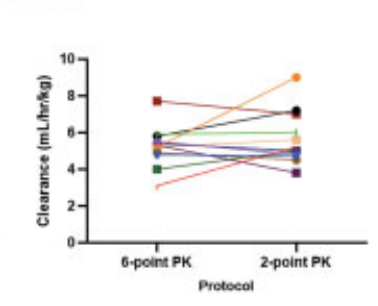

Chromogenic Assay

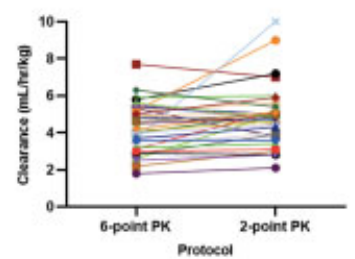

Non-o Blood Group

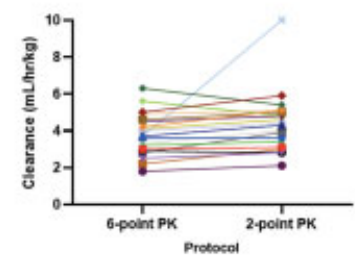

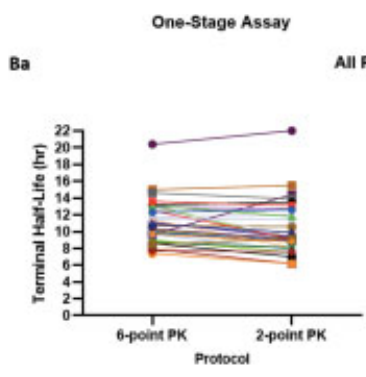

All Participants

Chromogenic Assay

Bb

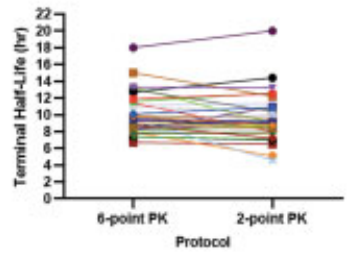

O Blood Group
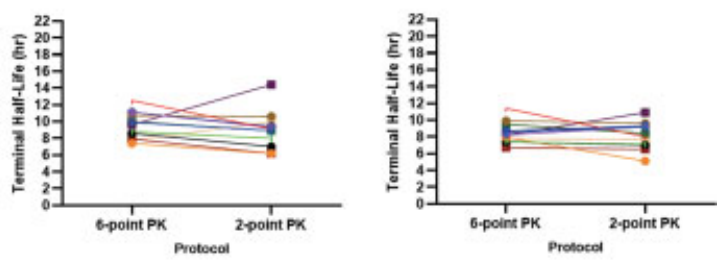

Bc

Non-o Blood Group

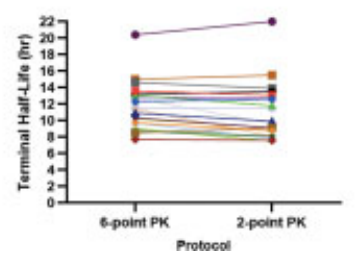

Fig. 2 Spaghetti plots showing, in the same subjects, the PK parameters of (A) clearance and (B) terminal half-life generated using the myPKFiT dosing tool and factor VIII:C levels from the washout, 6-sampling time point and the no-washout, single-clinic visit 2 time-point PK protocols. a: All participants $(n=35)$; b: subjects with O blood group $(n=12)$; c: subjects with non-O blood group ( $n=20)$. PK, pharmacokinetic.

Results for the PK parameter of $V_{\mathrm{ss}}$ and the agreement between the 6- and 2-point PK protocols are provided in the -Supplementary Material (-Supplementary Fig. 1 and -Supplementary Table 1, available in the online version). For all participants, the agreement between the two PK protocols for $V_{\mathrm{sS}}$ was moderate using FVIII:C levels determined with both the one-stage and chromogenic FVIII assays.

The degree of agreement between the 6- and 2-point PK protocols for the parameters of $\mathrm{Cl}$ and $t_{1 / 2}$ was greater for non-O blood group subjects than for $\mathrm{O}$ blood group subjects 
Table 2 Agreements between the washout, 6-sampling time point, and the no-washout, single-clinic visit 2 time-point PK protocols for the PK parameters of clearance and terminal half-life generated using the ADVATE myPKFiT dosing tool and factor VIII:C levels ${ }^{\mathrm{a}}$

\begin{tabular}{|l|l|l|l|}
\hline & \multirow{2}{*}{ PK parameter } & One-stage assay & Chromogenic assay \\
\cline { 3 - 4 } & & ICC $(95 \% \mathrm{Cl})$ & ICC $(95 \% \mathrm{Cl})$ \\
\hline \multirow{2}{*}{ All participants $(n=35)$} & $\mathrm{Cl}(\mathrm{mL} / \mathrm{h} / \mathrm{kg})$ & $0.73(0.52,0.85)$ & $0.54(0.25,0.74)$ \\
\cline { 2 - 4 } & $t_{1 / 2}(\mathrm{~h})$ & $0.84(0.70,0.91)$ & $0.80(0.63,0.89)$ \\
\hline \multirow{2}{*}{ O blood group $(n=12)$} & $\mathrm{Cl}(\mathrm{mL} / \mathrm{h} / \mathrm{kg})$ & $0.52(-0.04,0.84)$ & $0.35(-0.25,0.76)$ \\
\cline { 2 - 4 } & $t_{1 / 2}(\mathrm{~h})$ & $0.37(-0.23,0.76)$ & $0.37(-0.23,0.77)$ \\
\hline Non-O blood group $(n=20)$ & $\mathrm{Cl}(\mathrm{mL} / \mathrm{h} / \mathrm{kg})$ & $0.83(0.63,0.93)$ & $0.50(0.08,0.76)$ \\
\cline { 2 - 4 } & $t_{1 / 2}(\mathrm{~h})$ & $0.93(0.84,0.97)$ & $0.85(0.66,0.94)$ \\
\hline
\end{tabular}

Abbreviations: $\mathrm{Cl}$, clearance; PK, pharmacokinetic; $t_{1 / 2}$, terminal half-life.

Note: Data for 35/39 subjects were available for analysis: the ADVATE myPKFiT application was unable to generate PK parameters for the 2-point PKs of four subjects (two subjects had doses $<10 \mathrm{IU} / \mathrm{kg}$ which is outside the allowable range of $10-100 \mathrm{IU} / \mathrm{kg}$ for the dosing tool, one subject had technical issues with the FVIII:C samples and one subject did not have adequate documentation of the FVIII infusion prior to the clinic visit for the 2sampling time point PK study). Blood group status was not available for three subjects.

a Factor VIII:C determinations done in central laboratory.

(-Table 2, -Supplementary Table 1, available in the online version), perhaps reflecting the difference in $\mathrm{Cl}$ of FVIII and therefore circulating FVIII levels at different time points between $\mathrm{O}$ and non-O blood group subjects, or the smaller number of $\mathrm{O}$ blood group subjects. The degree of agreement between the two protocols for $V_{\text {ss }}$ was moderate for both $O$ and non-O blood group subjects.

The agreements between the 6- and 2-point protocols for PK parameters generated using the myPKFiT dosing tool and the FVIII:C levels measured in the local coagulation laboratories of the study sites ( - Supplementary Table 2, available in the online version) are very similar to those measured in the central laboratory (-Table 2 and -Supplementary Table 1, available in the online version).

\section{Comparison between One-Stage and Chromogenic FVIII:C Assays}

PK parameters calculated using the myPKFiT dosing tool were used to compare one-stage and chromogenic FVIII:C levels for both protocols (6- and 2-point). An almost perfect agreement was observed on both protocols when comparing $\mathrm{Cl}$ ( 0.84 and 0.91 , respectively) and $t_{1 / 2}$ ( 0.91 and 0.93 , respectively). $V_{\text {ss }}$ showed a substantial agreement ( 0.60 and 0.71 , respectively). The frequency distributions of $\mathrm{Cl}$ and $t_{1 / 2}$ calculated using the myPKFiT dosing tool from the 6-point and 2-point PK protocols (one-stage and chromogenic) are presented in - Fig. 3(A, B).

One-stage and chromogenic FVIII:C levels were compared for each sampling time point using data from the washout, 6-point PK protocol. FVIII:C levels with the chromogenic assay were significantly higher at early time points, 1 and 3 hours (by 17.8 and $9.0 \%$, respectively), but significantly lower at later time points, pre- and at 48 hours postinfusion (by 22.1 and $18.4 \%$, respectively) in comparison to the one-stage assay. The mean difference between the one-stage and chromogenic assay FVIII:C levels for the 9- and 24-hour time points was not statistically significantly different. These results are presented graphically in -Supplementary Fig. 2 (available in the online version).

\section{PK Parameters among Blood Groups}

PK parameters calculated with the myPKFiT dosing tool using both one-stage and chromogenic FVIII:C levels from the washout, 6-point PK protocol were compared between subjects who were $\mathrm{O}$ blood group and those who were non-O blood group (A, B, or AB). Subjects who were O blood group had significantly higher $\mathrm{Cl}$ values than subjects who were non-O blood group for both the one stage $(4.76 \pm 1.06 \mathrm{vs} .3 .80 \pm 1.33 \mathrm{~mL} / \mathrm{h} / \mathrm{kg}$, respectively; $p=0.03)$ and chromogenic $(4.95 \pm 1.25$ vs. $3.67 \pm 1.16$ $\mathrm{mL} / \mathrm{h} / \mathrm{kg}$, respectively; $p=0.004)$ FVIII:C assays. Reflecting these differences in $\mathrm{Cl}$ of infused FVIII, subjects who were $\mathrm{O}$ blood group had significantly lower $t_{1 / 2}$ values than subjects who were non-O blood group for both the one-stage $(9.9 \pm 1.8$ vs. $12.1 \pm 2.97$ hours, respectively; $p=0.02$ ) and chromogenic $(8.8 \pm 1.6$ vs. $11.0 \pm 2.6$ hours, respectively; $p=0.01)$ FVIII:C assays. Subjects who were O blood group had a shorter $\mathrm{tt} 1 \%$ when compared with subjects who were non-O blood group for both the one-stage ( $60.4 \pm 12.6$ vs. $74.1 \pm 20.0$ hours, respectively; $p=0.03$ ) and chromogenic ( $54.4 \pm 11.8$ vs. $68.7 \pm 17.9$ hours respectively; $p=0.01)$ FVIII:C assays. There were no statistically significant differences between subjects who were $O$ blood group and non- $O$ blood group for $V_{\text {ss }}$ derived from both FVIII:C assays.

\section{PK Parameters and VWF:Ag and VWF:pp Levels}

PK parameters calculated with the myPKFiT dosing tool using both the one-stage and chromogenic FVIII:C levels from the washout, 6-point PK protocol were compared with VWF:Ag levels. There were very strong negative correlations between VWF:Ag levels and $\mathrm{Cl}$ of infused FVIII derived from both the one-stage assay $(r=-0.74, p<0.0001)$ and the chromogenic assay $(r=-0.76, p<0.0001)$. Similarly, there were strong positive correlations between VWF:Ag levels and $t_{1 / 2}$ derived with both the one-stage assay $(r=0.69, p<0.0001)$ and the chromogenic assay $(r=0.76, p<0.0001)$. There was also a very strong positive correlation between VWF:Ag levels and tt $1 \%$ with both the one-stage assay $(r=0.72, p<0.0001)$ and the chromogenic assay FVIII:C levels $(r=0.77, p<0.0001)$. 


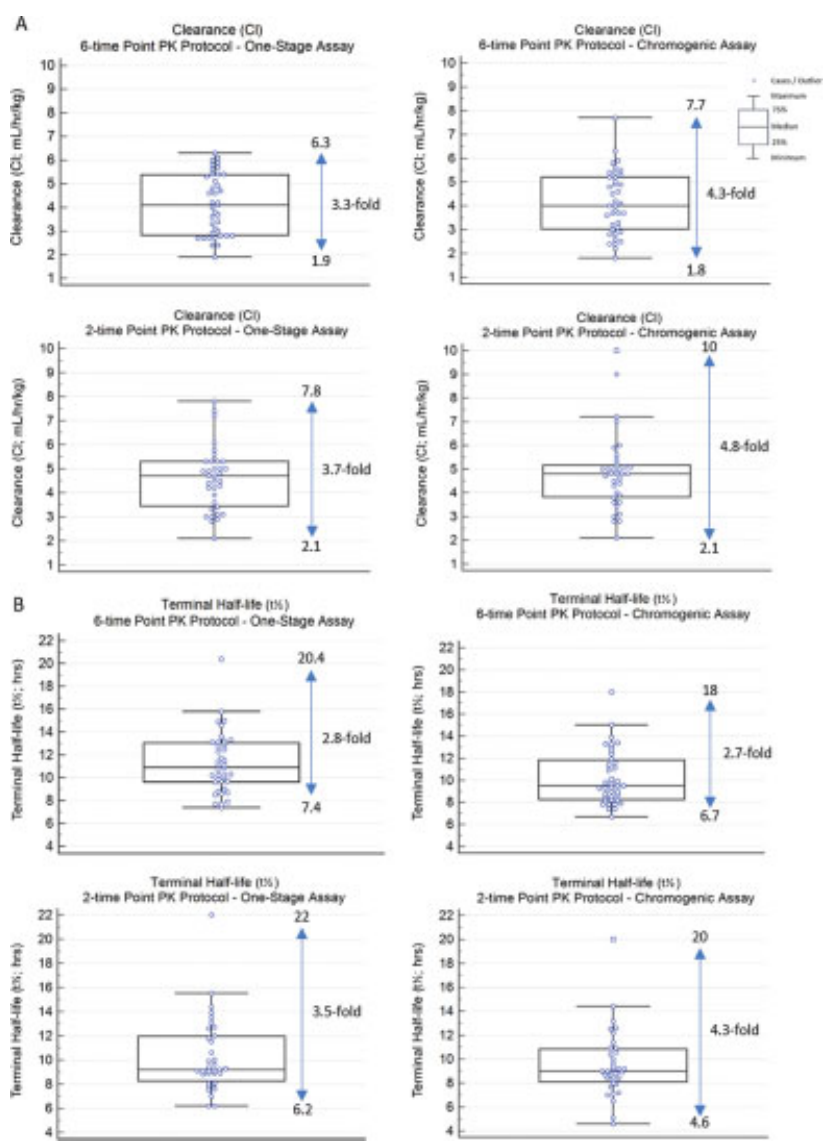

Fig. 3 Box and whisker plots of $(A)$ clearance $(\mathrm{Cl})$ and $(B)$ terminal half-life $\left(t_{1 / 2}\right)$ for one-stage and chromogenic FVIII:C assays $(n=39$ for 6 -point PK protocol; $n=35$ for 2-point PK protocol). The maximum and minimum values are given beside the plots, with the fold difference in the values as indicated.

There was a moderate negative correlation between VWF:Ag and $V_{\mathrm{ss}}$ derived from the one-stage assay $(r=-0.53$, $p=0.001)$ and a strong negative correlation with the chromogenic assay $(r=-0.64, p<0.0001)$. There were significant differences between VWF:Ag levels in subjects who were 0 blood group and non-O blood group $(p=0.002)$, with the $O$ group subjects $(0.72 \pm 0.16 \mathrm{IU} / \mathrm{dL})$ having lower VWF:Ag levels compared with the non-O blood group subjects $(1.08 \pm 0.37 \mathrm{IU} / \mathrm{dL})$. There was also a significant difference between VWF:pp/VWF:Ag ratios in subjects who were 0 blood group and those who were non-O blood group $(1.63 \pm 0.55$ and $1.19 \pm 0.54$, respectively, $p=0.031)$. This finding is consistent with higher $\mathrm{Cl}$ of VWF in O blood group patients.

The 6-point PK parameters $\mathrm{Cl}, t_{1 / 2}, \mathrm{tt} 1 \%$, and $V_{\mathrm{ss}}$ calculated with myPKFiT for both the one-stage and chromogenic FVIII:C levels were compared with VWF:pp levels and VWF:pp/VWF:Ag levels. No statistically significant correlations were found between the PK parameters and VWF:pp levels or VWF:pp/VWF:Ag ratios.

\section{PK Parameters and Age}

PK parameters calculated with the myPKFiT dosing tool using both the one-stage and chromogenic assay FVIII:C levels were compared with age. There were fair negative correla- tions between age and $\mathrm{Cl}$ derived from the one-stage ( $r=$ $-0.50, p=0.001)$ and chromogenic assays $(r=-0.49$, $p=0.002)$ and between age and $V_{\text {ss }}$ derived from the onestage $(r=-0.39, p=0.014)$ and chromogenic assays $(r=$ $-0.57, p=0.0001$ ). Reflecting these results, there were also moderate positive correlations between age and $t_{1 / 2}$ derived from the one-stage $(r=0.63, p<0.0001)$ and the chromogenic FVIII:C levels $(r=0.67, p<0.0001)$, as well as between age and tt $1 \%$ derived from the one-stage $(r=0.58$, $p=0.0001)$ and chromogenic assays $(r=0.62, p<0.0001)$.

\section{Discussion}

Several recent reports have documented the potential benefits of individualized prophylaxis regimens based on measured PK profiles of subjects with hemophilia A. ${ }^{7,21-23} \mathrm{~A}$ challenge to the implementation of PK-directed prophylaxis regimens in the past was that traditional PK studies in individuals with hemophilia were demanding as they required a long washout to achieve trough levels below the limit of quantitation of FVIII using conventional one-stage or chromogenic FVIII:C assays, followed by multiple postinfusion blood samples. Consequently, such PK studies were impractical from a clinical perspective. ${ }^{6}$ More recently, the development of PPK dosing tools have, with Bayesian forecasting, facilitated measurement of PK profiles without an obligatory washout and with sparse blood sampling. In a recent communication from the Scientific Standardization Committee of the ISTH, Iorio and colleagues recommended two to three sampling time points at least 12 hours apart, e.g., 4 to 8,16 to 28 , and 40 to 60 hours following infusion of a SHL FVIII concentrate; for extended half-life (EHL) FVIII concentrates, an additional sample obtained at 60 to 84 hours was recommended. The authors also stressed that the most informative sample is the one taken at 24 hours. $^{12}$ Although this is a marked improvement over the traditional washout, multiple blood sampling PK protocol advocated by the ISTH several years ago, this modified, no-washout PPK protocol still requires blood sampling at several outpatient clinic visits. This will either increase the number of nonroutine clinic visits or increase the time until PK results will be available (if blood samples are collected only during routine outpatient hemophilia comprehensive care clinic visits, generally every 6 to 12 months).

To the best of our knowledge, this is the first study to compare a no-washout, sparse, reverse sampling, singleclinic visit PK protocol (2-point PK protocol) to a washout, multiple clinic visit protocol (6-point PK protocol) as recommended in the past by the ISTH. ${ }^{4}$ The times of the blood draws in this study were chosen based on the myPKFiT User Manual, which states that a PK profile can be generated when the recommended minimum sampling conditions, one at 3 to 4 hours and the second at 24 to 32 hours postinfusion, are met $^{7,24}$; this also complies with the recent ISTH guidance published by Iorio and colleagues. ${ }^{12}$ Most importantly, we chose this 2-sampling time point protocol, in which the samples are taken in a reverse order of time, i.e., 24-hour sample first, followed by the 3-hour sample, based on the fact 
that sampling can be performed during a routine comprehensive care hemophilia clinic visit, which in our experience, if it includes medical, nursing, and physiotherapist reviews, generally lasts approximately 3 hours. Thus, this "reverse" sampling PK protocol is convenient for patients and their families, and practical for caregivers. We reasoned that if the results of our study yielded good comparability between a no-washout, single-clinic visit 2-point PK protocol and a more traditional washout, multiple clinic visit, 6-sampling time point PK protocol, the uptake of PK profiling of subjects with hemophilia A receiving prophylaxis with ADVATE using the myPKFiT PPK dosing tool at routine comprehensive care hemophilia clinic visits could be greatly enhanced.

Indeed, the results of this study are encouraging. We demonstrated, using data from the washout, 6-point PK protocol with FVIII:C levels measured using either the one-stage or chromogenic assays, that results obtained with the two-compartment PPK myPKFiT dosing tool and with the gold standard individualized PK program WinNonlin are comparable. We then determined that there was moderate to almost perfect agreement for all PK parameters, $\mathrm{Cl}, t_{1 / 2}$, and $V_{\mathrm{ss}}$, obtained with the washout, 6-point PK protocol and the no-washout, single out-patient hemophilia clinic visit, 2-point PK protocol using the one-stage and chromogenic assays done in the central laboratory. Results for $\mathrm{Cl}$ and $t_{1 / 2}$, the two PK parameters of greatest importance with regard to dosing for prophylaxis regimens, are highlighted in this study. Of note and of importance from a practical perspective, very similar results were obtained using the one-stage FVIII:C results reported by the local coagulation laboratories. Overall, we interpret these findings as positive from a clinical viewpoint, suggesting that the no-washout, single-clinic visit 2-point PK protocol analyzed with the myPKFiT PPK dosing tool generally yields a very good approximation of the PK profile for ADVATE infused into subjects with severe hemophilia A.

When the FVIII:C levels for each time point were compared between assays, the chromogenic assay showed higher levels at 1 and 3 hours postinfusion and lower levels for the pre- and 48-hour postinfusion samples. These findings are consistent with results reported by Morfini and colleagues, ${ }^{25}$ where it was shown that when FVIII:C levels are above $25 \mathrm{IU} / \mathrm{dL}$, the chromogenic assay yields higher FVIII:C levels than does the one-stage assay and lower FVIII:C levels than the one-stage assay when FVIII:C levels are below $25 \mathrm{IU} / \mathrm{dL}$. These differences did not impact in any significant way on the PK parameters of $\mathrm{Cl}$ or $t_{1 / 2}$, as detailed in this report.

Additional important findings from this study relate to the importance of baseline VWF:Ag levels on $\mathrm{Cl}$ of infused ADVATE which was significantly higher in subjects with lower VWF:Ag levels, resulting in lower $t_{1 / 2}$ values in these subjects. In parallel, $\mathrm{Cl}$ of infused ADVATE was significantly higher in subjects who were $O$ blood group as compared with non-O blood group subjects, reflecting the well-known fact that levels of VWF:Ag, the binding partner for FVIII in the circulation, are substantially lower in blood $O$ group subjects as compared with non-O blood group subjects. ${ }^{26-28} \mathrm{In} \mathrm{fact,} \mathrm{in}$ a recent publication, Lunghi et al showed that there is a higher intercompartment $\mathrm{Cl}$, as well as transfer rates between central and peripheral compartments, in $\mathrm{O}$ blood group subjects when compared with non-O blood group subjects. ${ }^{28}$ These differences between $\mathrm{O}$ and non-O blood group subjects might have had an impact on the agreement between the 6- and the 2-point PK protocols, since our results showed that the non- $\mathrm{O}$ group had a greater level of agreement than the $\mathrm{O}$ group.

This association between blood groups and VWF:Ag on both $\mathrm{Cl}$ and $t_{1 / 2}$ of infused SHL and EHL FVIII concentrates has been documented in several studies ${ }^{26,27,29,30}$ and reflects the fact that clearance of infused FVIII is driven by clearance of its binding partner, VWF:Ag. The importance of these associations should not be underestimated. The approximate three- to five-fold difference in $\mathrm{Cl}$ and $t_{1 / 2}$ between inhibitornegative subjects with severe hemophilia A observed in this study, and also reported by others, ${ }^{25,31,32}$ exceeds the approximate 1.5 -fold difference in $\mathrm{Cl}$ and $t_{1 / 2}$ observed between EHL and SHL FVIII concentrates, ${ }^{33}$ and should be taken into consideration when recommending individual prophylaxis regimens for subjects with severe hemophilia A. This study also showed a negative correlation between $V_{s s}$ and VWF:Ag, which could be due to the fact that VWF retains FVIII inside the plasma compartment; this may reduce FVIII in the extravascular spaces, creating a decrease in $V_{\text {ss. }}$.

Another relationship of note relates to the effect of age on $\mathrm{Cl}$ and $t_{1 / 2}$ of infused FVIII. In this study, as in some other reported studies, ${ }^{20,34,35} \mathrm{Cl}$ of infused FVIII (e.g., ADVATE) was higher in younger subjects. As subjects age, there is a change in body size and fluid volumes that impacts on the $V_{\text {ss }}$ and therefore would decrease $\mathrm{Cl}$ and increase $t_{1 / 2}$ of FVIII. ${ }^{36} \mathrm{We}$ believe that this effect is most marked for very young boys with hemophilia, since a recent study comparing PK parameters of two EHL FVIII concentrates in inhibitor-negative adolescent males with hemophilia A failed to document an age effect on $\mathrm{Cl}$ and $t_{1 / 2} \cdot{ }^{26}$ It appears likely, therefore, that PK profiles will not change substantially after adolescence and do not need to be repeated at regular intervals in such age groups (adolescents and adults). It is prudent, however, to consider repeat PK testing when switches are made from one FVIII concentrate to another and at an interval of every few years in young boys with hemophilia $\mathrm{A}<12$ years of age, and especially $<6$ years of age.

This study has some limitations. First is the number of samples collected for PK analysis. Some patients might not robustly fit a two-compartment model based on six samples; however, the collection of more blood samples has ethical and physiological limitations in pediatric patients that would make it challenging to obtain more blood samples. Therefore, the 6-point PK protocol based on the ISTH guidelines was used as a reference, rather than a traditional PK study based on more blood samples. ${ }^{4,5}$ Second is the premise that there is negligible inter-occasion variability in our 2sample reverse sampling strategy as it requires two different infusions. The fact that the infusion doses varied within the 2-point PK protocol precluded our ability to directly compare the $\mathrm{tt} 1 \%$ between the 6 - and 2-point PK protocols and this comparison is therefore not made in this study. Third, this 
study depends on patients/families taking the correct dose of factor and reporting the exact time of infusions. This is particularly important as we were indeed not able to perform the 2-sample PK protocol on three subjects due to incorrect doses (outside the myPKFiT ranges), or due to inadequate documentation of prior infusions. Fourth, the product-specific myPKFiT tool for ADVATE, only, was used in the determination of PK parameters in this study; in future studies, other PPK programs that are not product-specific, such as the WAPPS-Hemo (Web-based Application for the Population Pharmacokinetic Service Hemophilia), ${ }^{37}$ could be utilized. Fourth, as WinNonlin requires more than five samples to be able to perform a PK profile, it was not possible to compare the results from the 2-point PK from the myPKFiT dosing tool with those from the WinNonlin program. Finally, and most importantly, our study excluded subjects with several a priori determined comorbidities (e.g., presence of a recent bleed, laboratory evidence of clinically significant active hepatitis) that potentially could impact the clearance of infused FVIII; this represents a "best case" baseline scenario and it is likely that in a "real-world" setting, observed PK profiles may, in some cases, reflect presence of comorbidities, recognized or even unrecognized. However, it is very important that baseline PK profiles are obtained with subjects in a nonbleeding state and free of active systemic infection; when such studies are performed as part of a switch from one FVIII concentrate to another, the PK study should only be performed after the subject has received a reasonable number of infusions of the new FVIII concentrate and not at the time of the first infusion.

\section{Conclusion}

In summary, this study, conducted in 39 inhibitor-negative subjects with severe hemophilia $\mathrm{A}$ (median age of 11 years, and with $71.8 \%$ of cases $\leq 18$ years of age), demonstrated that a no-washout, single (3 hour) outpatient hemophilia clinic visit, 2-sampling time point PK protocol analyzed with the ADVATE myPKFiT PPK dosing tool generates PK parameters, $\mathrm{Cl}, t_{1 / 2}$, and $V_{\mathrm{ss}}$, with sufficient accuracy compared with a washout, 6-sampling time point, 3 clinic visit PK protocol. Thus, a 2-sampling time point PK protocol is very attractive for routine clinical use associated with comprehensive care hemophilia assessment visits. Results from the initial 2sampling time point PK study, performed with subjects in a nonbleeding state on prophylaxis after at least a few infusions of the FVIII concentrate under study, can be used to guide design of individual prophylaxis regimens. Additionally, of importance, the results can inform to the timing of future PK sampling time points at times convenient for subjects with hemophilia and their families and caregivers leading to more robust estimates of PK profiles. Although there remains controversy amongst health care providers involved with the assessment and care of persons with hemophilia regarding the clinical value of PK studies, we believe that PPK programs such as the ADVATE myPKFiT dosing tool and others (e.g., the WAPPS/Hemo Program ${ }^{37}$ ) are important tools for optimization of prophylaxis regimens at the individual patient level with the goal of preventing long-term hemophilic arthropathy.

\section{What is known about this topic?}

- Traditional pharmacokinetic (PK) assessments are demanding for persons with hemophilia A. They require a washout period and multiple blood sampling time points over a 72-hour period following infusion of a standard half-life FVIII clotting factor concentrate such as ADVATE.

- Population PK (PPK) models have been developed for persons with hemophilia A.

\section{What does this paper add?}

- PPK parameters were obtained with a traditional washout, 6-sampling time point PPK and a more practical, no-washout, reverse 2-sampling time point, single-visit PPK protocol in persons with severe hemophilia A using the ADVATE myPKFiT dosing tool and were compared.

- The "user-friendly" 2-point PPK protocol was found to generate PK parameters with sufficient accuracy. Thus, 2-point PPK results can be used to guide prophylaxis regimens in persons with severe hemophilia A receiving ADVATE.

\section{Funding}

This research was funded by an Investigator Initiated Research Grant from Baxalta U.S. Inc., a Takeda company, Lexington, Massachusetts (Grant ID number: \#IIRH1527025), with The Hospital for Sick Children as the sponsor site.

\section{Conflict of Interest}

V.B. has received consultancy and speaker fees from Amgen, Bayer, Bioverativ/Sanofi, Novo Nordisk, Pfizer, Roche, Shire/Takeda, and Spark Therapeutics. He is recipient of research grants from Bioverativ/Sanofi, Bayer, Novo Nordisk, and Shire, and serves as a member of Data Safety Monitoring Boards for Octapharma and Shire/Takeda. He is also the Chair of a nonprofit organization, the International Prophylaxis Study Group (IPSG). J.B. has received consultancy and/or speaker fees from Bayer, LFB, Novo Nordisk, Pfizer, Roche, Shire/Takeda, and Sobi. M.C. reports having received research support from Bayer, Bioverativ/Sanofi, CSL Behring, Novo Nordisk, Octapharma, Pfizer, and Shire/Takeda. He has also received honoraria for speaking/participating in advisory boards from Bayer, Bioverativ/Sanofi, Biotest, CSL Behring, Grifols, LFB, Novo Nordisk, Octapharma, Pfizer, Roche, and Shire/Takeda. D.L. has received research support from Biomarin, Bayer, Bioverativ/Sanofi, CSL-Behring, and Octapharma. M.M. has acted as a paid consultant to Bayer, Baxter, Novo Nordisk, and Pfizer for participation in 
advisory boards, and has received speaker fees for educational events organized by CSL Behring, Biotest, Bayer, Kedrion, Novo Nordisk, Sobi, and Octapharma. He has received research grant support from Pfizer. The other authors report no conflicts of interest to declare.

\section{References}

1 Ljung R, Gretenkort Andersson N. The current status of prophylactic replacement therapy in children and adults with haemophilia. Br J Haematol 2015;169(06):777-786

2 Collins PW, Blanchette VS, Fischer K, et al; rAHF-PFM Study Group. Break-through bleeding in relation to predicted factor VIII levels in patients receiving prophylactic treatment for severe hemophilia A. J Thromb Haemost 2009;7(03):413-420

3 Björkman S, Folkesson A, Jönsson S. Pharmacokinetics and dose requirements of factor VIII over the age range 3-74 years: a population analysis based on 50 patients with long-term prophylactic treatment for haemophilia A. Eur J Clin Pharmacol 2009;65 (10):989-998

4 Lee M, Morfini M, Schulman S, Ingerslev J. The Factor VIII/Factor IX Scientific and Standardization Committee of the International Society for Thrombosis and Haemostasis: the design and analysis of pharmacokinetic studies of coagulation factors. 2001. Accessed February 11, 2021 at: https://cdn.ymaws.com/www.isth.org/resource/group/d4a6f49a-f4ec-450f-9e0f-7be9f0c2ab2e/official_communications/fviiipharmaco.pdf

5 Morfini M, Lee M, Messori A. The design and analysis of half-life and recovery studies for factor VIII and factor IX. Factor VIII/Factor IX Scientific and Standardization Committee of the International Society for Thrombosis and Haemostasis. Thromb Haemost 1991; 66(03):384-386

6 Collins PW, Fischer K, Morfini M, Blanchette VS, Björkman S; International Prophylaxis Study Group Pharmacokinetics Expert Working Group. Implications of coagulation factor VIII and IX pharmacokinetics in the prophylactic treatment of haemophilia. Haemophilia 2011;17(01):2-10

7 Mingot-Castellano ME, Parra R, Núñez R, Martorell M. Improvement in clinical outcomes and replacement factor VIII use in patients with haemophilia A after factor VIII pharmacokineticguided prophylaxis based on Bayesian models with myPKFiT ${ }^{\circledR}$. Haemophilia 2018;24(05):e338-e343

8 Sheiner LB, Rosenberg B, Marathe VV. Estimation of population characteristics of pharmacokinetic parameters from routine clinical data. J Pharmacokinet Biopharm 1977;5(05):445-479

$9 \mathrm{Yu}$ JK, Iorio A, Edginton AN; WAPPS co-investigators. Using pharmacokinetics for tailoring prophylaxis in people with hemophilia switching between clotting factor products: a scoping review. Res Pract Thromb Haemost 2019;3(03):528-541

10 Ette EI, Williams PJ. Population pharmacokinetics I: background, concepts, and models. Ann Pharmacother 2004;38(10):1702-1706

11 Björkman S, Oh M, Spotts G, et al. Population pharmacokinetics of recombinant factor VIII: the relationships of pharmacokinetics to age and body weight. Blood 2012;119(02):612-618

12 Iorio A, Blanchette V, Blatny J, Collins P, Fischer K, Neufeld E. Estimating and interpreting the pharmacokinetic profiles of individual patients with hemophilia A or B using a population pharmacokinetic approach: communication from the SSC of the ISTH. J Thromb Haemost 2017;15(12):2461-2465

13 Hazendonk HCAM, van Moort I, Mathôt RAA, et al; OPTI-CLOT study group. Setting the stage for individualized therapy in hemophilia: What role can pharmacokinetics play? Blood Rev 2018;32(04):265-271

14 Toutain PL, Bousquet-Mélou A. Plasma terminal half-life. J Vet Pharmacol Ther 2004;27(06):427-439

15 Blanchette VS, Shapiro AD, Liesner RJ, et al; rAHF-PFM Clinical Study Group. Plasma and albumin-free recombinant factor VIII: pharmacokinetics, efficacy and safety in previously treated pediatric patients. J Thromb Haemost 2008;6(08):1319-1326

16 Tarantino MD, Collins PW, Hay CR, et al; RAHF-PFM Clinical Study Group. Clinical evaluation of an advanced category antihaemophilic factor prepared using a plasma/albumin-free method: pharmacokinetics, efficacy, and safety in previously treated patients with haemophilia A. Haemophilia 2004;10(05):428-437

17 Fleiss JL, Cohen J. The equivalence of weighted kappa and the intraclass correlation coefficient as measures of reliability. Educ Psychol Meas 1973;33:613-619

18 Landis JR, Koch GG. The measurement of observer agreement for categorical data. Biometrics 1977;33(01):159-174

19 Akoglu H. User's guide to correlation coefficients. Turk J Emerg Med 2018;18(03):91-93

20 Björkman S, Blanchette VS, Fischer K, et al; Advate Clinical Program Group. Comparative pharmacokinetics of plasma- and albumin-free recombinant factor VIII in children and adults: the influence of blood sampling schedule on observed age-related differences and implications for dose tailoring. J Thromb Haemost 2010;8(04):730-736

21 Pasca S, Milan M, Sarolo L, Zanon E. PK-driven prophylaxis versus standard prophylaxis: when a tailored treatment may be a real and achievable cost-saving approach in children with severe hemophilia A. Thromb Res 2017;157:58-63

22 Nagao A, Yeung CHT, Germini F, Suzuki T. Clinical outcomes in hemophilia A patients undergoing tailoring of prophylaxis based on population-based pharmacokinetic dosing. Thromb Res 2019; 173:79-84

23 Stemberger M, Kallenbach F, Schmit E, et al. Impact of adopting population pharmacokinetics for tailoring prophylaxis in haemophilia A patients: a historically controlled observational study. Thromb Haemost 2019;119(03):368-376

24 MyPKFiT for Healthcare Professionals-User Manual. 3th ed. Bannockburn, IL: Baxalta U.S. Inc., an entity of Shire PLC; 2019:29

25 Morfini M, Cinotti S, Bellatreccia A, et al; PMReFacto-AICE Study Group. A multicenter pharmacokinetic study of the B-domain deleted recombinant factor VIII concentrate using different assays and standards. J Thromb Haemost 2003;1(11):2283-2289

26 Carcao MD, Chelle P, Clarke E, et al. Comparative pharmacokinetics of two extended half-life FVIII concentrates (Eloctate and Adynovate) in adolescents with hemophilia A: is there a difference? J Thromb Haemost 2019;17(07):1085-1096

27 O'Donnell J, Laffan MA. The relationship between ABO histoblood group, factor VIII and von Willebrand factor. Transfus Med 2001;11(04):343-351

28 Lunghi B, Bernardi F, Martinelli N, et al. Functional polymorphisms in the LDLR and pharmacokinetics of Factor VIII concentrates. J Thromb Haemost 2019;17(08):1288-1296

29 Fischer K, Pendu R, van Schooten CJ, et al. Models for prediction of factor VIII half-life in severe haemophiliacs: distinct approaches for blood group O and non-O patients. PLoS One 2009;4(08): e6745

30 Kepa S, Horvath B, Reitter-Pfoertner S, et al. Parameters influencing FVIII pharmacokinetics in patients with severe and moderate haemophilia A. Haemophilia 2015;21(03):343-350

31 Barnes C, Lillicrap D, Pazmino-Canizares J, et al. Pharmacokinetics of recombinant factor VIII (Kogenate-FS ${ }^{\circledR}$ ) in children and causes of inter-patient pharmacokinetic variability. Haemophilia 2006; 12(04):40-49

32 Collins PW, Björkman S, Fischer K, et al. Factor VIII requirement to maintain a target plasma level in the prophylactic treatment of severe hemophilia A: influences of variance in pharmacokinetics and treatment regimens. J Thromb Haemost 2010;8(02): 269-275

33 Gringeri A, Wolfsegger M, Steinitz KN, Reininger AJ. Recombinant full-length factor VIII (FVIII) and extended half-life FVIII products in prophylaxis-new insight provided by pharmacokinetic modelling. Haemophilia 2015;21(03):300-306 
1336 PK Profiles Using Sparse Sampling: Hemophilia A Blanchette et al.

34 Carlsson M, Berntorp E, Björkman S, Lethagen S, Ljung R. Improved cost-effectiveness by pharmacokinetic dosing of factor VIII in prophylactic treatment of haemophilia A. Haemophilia 1997;3(02):96-101

35 Matucci M, Messori A, Donati-Cori G, et al. Kinetic evaluation of four factor VIII concentrates by model-independent methods. Scand J Haematol 1985;34(01):22-28
36 Björkman S. Comparative pharmacokinetics of factor VIII and recombinant factor IX: for which coagulation factors should halflife change with age? Haemophilia 2013;19(06):882-886

37 Hajducek DM, Chelle P, Hermans C, et al. Development and evaluation of the population pharmacokinetic models for FVIII and FIX concentrates of the WAPPS-Hemo project. Haemophilia 2020;26(03):384-400 